\title{
UTILIDAD DE MARCADORES SÉRICOS DEL CARCINOMA RENAL
}

\author{
J. RUBIO BRIONES, I. IBORRA JUAN, E. AZNAR*, M.A. CLIMENT**, \\ J.A. LÓPEZ-GUERRERO***, J. MAÍQUEZ*, J.L. MONRÓS LLISO, \\ J. CASANOVA RAMÓN-BORJA, R. DUMONT MARTÍNEZ, J.V. RICÓS TORRENT, \\ E. SOLSONA NARBÓN
}

\begin{abstract}
Servicio Urología. *Servicio de Laboratorio. **Servicio de Oncología Médica. ***Biología Molecular. Instituto Valenciano de Oncología. Valencia.

Actas Urol Esp. 28 (5): 381-386, 2004
\end{abstract}

\section{RESUMEN}

UTILIDAD DE MARCADORES SÉRICOS DEL CARCINOMA RENAL

FUNDAMENTO: Seleccionar un grupo de marcadores tumorales útiles en el carcinoma renal (CR) para su ulterior estudio y desarrollo.

MÉTODOS: Determinación seriada en suero de 21 pacientes con CR de los siguientes marcadores: VSG, PCR, hemograma y recuento diferencial leucocitario (RDL), bioquímica estándar, ß2-microglobulina, CEA, CA 12.5, CA 50, CA 15.3, ferritina, interleukina-6, receptor de la interleukina-2, factor de necrosis tumoral $\alpha$ y antígeno tisular polipeptídico.

RESULTADOS: Diferentes elementos dentro del RDL tuvieron relación con la presencia de clínica, con el tamaño, estadio patológico y la progresión tumoral. Se observa un incremento estadísticamente significativo de los valores de la $\beta-2$ microglobulina y del RIL-2 en el momento de la progresión, así como un aumento con tendencia a la significación de los valores de la PCR y las fosfatasas alcalinas. La $\beta-2$ microglobulina y el RIL-2 decrecen significativamente al tratar la progresión.

CONCLUSIONES: Seguiremos determinando el hemograma, RDL y bioquímica estándar, la PCR, la ferritina, la $\beta-2$ microglobulina, y el RIL-2 exclusivamente con el objeto de delimitar su utilidad en el seguimiento de los pacientes con CR, desestimando la determinación del resto de marcadores testados.

PALABRAS CLAVE: Carcinoma renal. Marcador. Suero.

\section{ABSTRACT \\ USEFULNESS OF SERUM MARKERS IN RENAL CELL CARCINOMA}

OBJECTIVES: To select a group of useful serum markers in renal cell carcinoma (RCC) with investigational purpose in future.

MATERIAL AND METHODS: Periodic determination in serum of 21 RCC patients of the following markers: EGR, RPC, hemogram and leucocyte differential count (LDC), standard biochemist parameters, $\beta-2$ microglobuline, CEA, CA 12.5, CA 50, CA 15.3, ferritin, interleukin-6, serum interleukin-2 receptor, TNF$\alpha$ and TPSA.

RESULTS: Different elements within the LDC had relations with the presence of symptoms/signs, tumour size, pathological stage and disease progression. There was a significant increase of $\beta-2$ microglobuline and sIL-2 receptor when disease progressed, as well as a similar statistical trend with RPC and alkaline phosphatases. $\beta-2$ microglobuline and sIL-2 receptor also decreased after treatment of the disease progression.

CONCLUSIONS: We will keep analysing hemogram, LDC and standard byochemics, RPC, ferritin, $\beta-2$ microglobuline and sIL-2 receptor only with investigational purposes, obviating the determination of the rest of the tested markers.

KEY WORDS: Renal cell carcinoma. Marker. Serum. 
A sí como en otros tumores urológicos, se ha generalizado el uso de marcadores séricos para su diagnóstico específico (ej. PSA en el carcinoma prostático) o para su seguimiento (ej. $\alpha \mathrm{FP}$ o $\beta \mathrm{HCG}$ en ciertos tumores testiculares), en el manejo del carcinoma renal (CR) se echa en falta un marcador tumoral específico que permita completar el manejo del mismo. Muchos grupos han testado diferentes marcadores en el seguimiento del CR, muchas veces de manera anecdótica y sin refrendarse posteriormente por otros grupos o en estudios prospectivos. Todos los marcadores estudiados se pueden agrupar en tres grandes grupos.

En primer lugar se engloban todos aquellos marcadores séricos inespecíficos -utilizados muchos de ellos en el seguimiento clínico de muchas enfermedades sistémicas y tumorales-, dentro de los cuales encontramos las proteínas reactantes de fase aguda como la proteína $\mathrm{C}$ reactiva $(\mathrm{PCR})^{1}$, la velocidad de sedimentación (VSG), la hemoglobina, las fosfatasas alcalinas ${ }^{2,3}$, el antígeno polipeptídico tisular específico ${ }^{4}$ (TPSA), las proteínas involucradas en el metabolismo del hierro, sobre todo la ferritina ${ }^{5-7}$ y la $\beta-2$ microglobulina ${ }^{8}$.

En segundo lugar se englobarían determinadas glicoproteínas que se han usado como marcadores tumorales en otro tipo de tumores, y que, atendiendo a la embriología común de muchas de las líneas celulares de distintos órganos, han sido también estudiadas en el CR. En este grupo se engloban; CEA, CA-50, CA 15-3, CA 509 y el factor de necrosis tumoral $\alpha(\mathrm{TNF} \alpha)$.

En tercer lugar estarían los marcadores relacionados con la respuesta inmune; entre los de este grupo destacan la interleukina- $6^{10}$ y el receptor para la interleukina- $2^{11}$.

El marcador tumoral ideal ha de cumplir los siguientes enunciados: 1) ha de ser segregado exclusivamente por las células tumorales de un determinado tejido; 2) ha de poder ser detectado desde el momento en que dicho tumor es biológicamente activo; 3) ha de tener una relación positiva con el estadio del tumor; 4) ha de detectar la progresión del tumor; 5) ha de reflejar la respuesta al tratamiento aplicado; y 6) ha de predecir el pronóstico del paciente.

Del gran número de marcadores séricos propuestos a lo largo de las dos últimas décadas en el CR, ninguno de ellos ha cumplido los 6 puntos referidos previamente. Además, prácticamente todos se han valorado de una forma retrospectiva y descriptiva, no reproduciéndose la mayoría de ellos en otras series.

Nuestro objetivo en este trabajo ha sido testar de una forma no randomizada y con intención descriptiva y analítica la utilidad de una serie de marcadores propuestos en la literatura en un grupo heterogéneo y no seleccionado de pacientes con $\mathrm{CR}$, con la intención de escoger para su uso en un futuro aquellos marcadores que apuntaran utilidad clínica en el manejo de este impredecible tumor.

\section{MATERIAL Y MÉTODOS}

Entre marzo de 2000 y marzo de 2002 se les solicitó a los pacientes diagnosticados con anatomía patológica de CR de células claras su autorización para congelar suero para su uso en investigación.

La extracción de suero en los casos nuevos se realizó antes del tratamiento quirúrgico o inmunoterápico. En caso de progresión de la enfermedad, se les repetía la extracción previa a cualquier actitud terapéutica. En casos sin progresión, la analítica se repetía cada 6 meses y con fines estadísticos; sólo se tuvo en cuenta la última.

Se desestimaron del estudio aquellos pacientes con diagnóstico previo o ulterior de un segundo tumor o de una enfermedad sistémica que pudiera alterar los parámetros séricos estudiados (ej. diabetes mellitus, hepatopatías, etc.).

Se obtuvieron $20 \mathrm{ml}$ de sangre de cada paciente, de la que tras centrifugación a $1.200 \mathrm{~g}$ se separaron $4 \mathrm{ml}$ en alícuotas, congelándolas a $25^{\mathrm{o}}$ con la finalidad de conseguir una seroteca de estudio para las pruebas especiales y las repeticiones analiticas necesarias.

El resto de suero y sangre se utilizó de forma rutinaria para determinar los valores séricos del calcio (Ca) y fósforo (P), lactato deshidrogenasa (LDH), proteínas totales (PT) y bilirrubina en un analizador Alcyon (Abbott). Los valores de CEA, CA 12.5, CA 15.3, $\beta-2$ microglobulina y ferritina se determinaron mediante un inmunoensayo de micropartículas (MEIA) con un analizador AXYM (Abbott). Los resultados del hemograma y del RDL se determinaron en un analizador Cell-Dyn 3700 de Abbott. 
Como pruebas especiales efectuamos las determinaciones de la interleukina- 6 con un enzimoinmunoensayo (ELISA) ICN. El receptor de la interleukina-2 (RIL-2) y el factor de necrosis tumoral $\alpha(\mathrm{TNF} \alpha)$ se obtuvieron mediante un ELISA Immunotech. El antígeno tisular polipeptídico (TPA) con un método inmunoradiométrico (IRMA), la eritropoyetina (EPO) con un método enzimoinmunométrico y el antígeno carbohidratado Ca 50 con IRMA RIA-gnost.

La analítica estándar (no realizada específicamente por este estudio) en nuestro Centro consta de hemograma y RDL y los parámetros bioquímicos de glucosa, urea, creatinina, Na, K, ALT, AST, GGT, y fosfatasas alcalinas. Las unidades y valores de referencia del resto de parámetros estudiados quedan reflejados en la Tabla I.

El análisis estadístico se llevó a cabo con el objeto de determinar si los datos patológicos y clínicos se relacionaban con los datos séricos. Aplicamos el test del Chi cuadrado entre variables cualitativas y el test $\mathrm{T}$ de Student para comprobar la relación entre variables cualitativas y cuantitativas. Se asume la significación estadística con un $\mathrm{p}<0,05$ para todos los tests. Todo ello se realizó con el paquete estadístico SPSS versión 10.0 .

\section{TABLA I}

MARCADORES TUMORALES UTILIZADOS FUERA DE LA ANALÍTICA ESTÁNDAR EN NUESTRO CENTRO (UNIDADES Y VALORES DE REFERENCIA)

\begin{tabular}{|l|c|c|}
\hline Marcador & Unidades & Valores referencia \\
\hline CEA & $\mathrm{ng} / \mathrm{ml}$ & $<5$ \\
\hline CA 12.5 & $\mathrm{U} / \mathrm{ml}$ & $<35$ \\
\hline CA 15.3 & $\mathrm{U} / \mathrm{ml}$ & $<40$ \\
\hline CA 19.9 & $\mathrm{U} / \mathrm{ml}$ & $<25$ \\
\hline PSA & $\mathrm{ng} / \mathrm{ml}$ & $<4$ \\
\hline Bmicroglobulina & $\mu \mathrm{g} / \mathrm{ml}$ & $<3400$ \\
\hline Ferritina & $\mathrm{ng} / \mathrm{ml}$ & $\begin{array}{c}\text { Hombres }<20-<320 \\
\text { Mujeres }>6-<282\end{array}$ \\
\hline CA50 & $\mathrm{U} / \mathrm{ml}$ & $<24$ \\
\hline TPSA & $\mathrm{U} / \mathrm{ml}$ & $<75$ \\
\hline FNT & $\mathrm{pg} / \mathrm{ml}$ & $<20$ \\
\hline IL6 & $\mathrm{pg} / \mathrm{ml}$ & $<4$ \\
\hline RIL-2 & $\mathrm{pg} / \mathrm{ml}$ & $<80$ \\
\hline EPO & $\mathrm{mU} / \mathrm{mL}$ & $2,6-34$ \\
\hline
\end{tabular}

\section{RESULTADOS}

Se le extrajo suero a un total de treinta y siete pacientes; 13 pacientes se desestimaron para el estudio por presentar un segundo tumor (3 casos), patologías sistémicas tipo diabetes o hepatopatías (9 casos) o las dos eventualidades (1 caso). En 3 casos se perdió la muestra por problemas técnicos. Al final fueron seleccionados 21 pacientes, con una media de seguimiento de 27,9 meses.

Enumeramos a continuación exclusivamente las relaciones entre los datos séricos y los datos clínicos o patológicos estadísticamente significativas y aquellas que presentan tendencia a la significación y que, debido al corto número de casos, podrían tener utilidad en caso de un mayor número de casos estudiados.

Diez pacientes $(47,6 \%)$ fueron hallazgos incidentales frente a $11(52,4 \%)$, que presentaron previamente alguna manifestación clínica. Dentro del grupo sintomático, se objetivó un mayor número de leucocitos (a expensas de los neutrófilos) respecto al grupo de incidentales $(p=0,046)$ y una tendencia a la significación estadística de un mayor número de plaquetas y LDH. Curiosamente, en el grupo de los tumores hallados incidentalmente, también se objetivó una tendencia a la significación estadística de unos indices mayores de los marcadores tumorales CA19-9 y CA50 y del TPSA y unas tasas significativamente mayores de las cifras de CEA $(\mathrm{p}=0,025)$ respecto a los tumores con clínica.

Los tumores $\leq 7 \mathrm{~cm}(53 \%)$ presentaban mayor número de linfocitos que los mayores de $7 \mathrm{~cm}$ (47\%), aunque sin llegar a significación estadística. El estadio patológico no fue posible determinarlo en 2 pacientes diseminados diagnosticados mediante punción biópsica; del resto, 8 pacientes presentaron tumores organoconfinados (pT1pT2) y 11 extrarrenales (pT3-pT4). Los pacientes con estadio pT3-pT4 tenían un número de monocitos y CA125 significativamente mayor $(\mathrm{p}=0,011$ y $\mathrm{p}=0,021$ respectivamente), y unos niveles de glucosa significativamente menor $(\mathrm{p}=0,003)$ que los organoconfinados. El grado no se objetivó en 2 pacientes; del resto, todos fueron grado II excepto un grado I y otro grado III.

En 4 pacientes se objetivó infiltración ganglionar y 5 pacientes $(23,8 \%)$ presentaban metástasis a distancia en el momento del diagnóstico frente a 16 MO (76,2\%). En los tumores metastáticos de inicio, se objetivó unos valores más bajos 
de hemoglobina y leucocitos y unos valores más altos de $\beta-2$ microglobulina respecto a los no metastáticos.

Siete pacientes $(33 \%)$ presentaron progresión de la enfermedad (local y/o metastásica) en algún momento de su evolución. Cuatro pacientes (20\%) murieron por progresión de la enfermedad y 17 permanecen vivos, de los cuales 5 tienen enfermedad presente en el momento de la última revisión. Aquellos pacientes que no progresaron presentaban inicialmente un mayor número de neutrófilos, aunque sin significación estadística. Los niveles iniciales de ferritina fueron estadísticamente más elevados en los pacientes que progresaron $(\mathrm{p}=0,00)$ y se objetivó una tendencia a la significación con los niveles de RIL-2 inicialmente elevados.

En aquellos pacientes en los que hubo progresión, se objetivó una elevación estadísticamente significativa por encima de sus valores de normalidad de la $\beta-2$ microglobulina y del RIL-2, y una tendencia a incrementarse de los valores de la PCR y las fosfatasas alcalinas junto a un descenso del número de plaquetas (Tabla I). Estos valores tienden a equipararse a los correspondientes en el momento del diagnóstico tras el tratamiento de la progresión.

\section{DISCUSIÓN}

Dentro de lo que se considera una analítica estándar encontramos una serie de valores que nos pueden "orientar" en cuanto a la agresividad del cuadro. Se han realizado estudios pronósticos usando los valores de una analítica estándar: hemoglobina, albúmina, calcio, lactatodeshidrogenasa (LDH), fosfatasas alcalinas; tienen valor pronóstico en el estudio univariado, pero tan solo la hemoglobina, junto con el performace status (ECOG) del paciente retienen su valor pronóstico en el estudio multivariado realizado por Citterio y cols, ${ }^{12}$ estableciendo con ambos tres grupos pronósticos con diferencias estadísticamente significativas en supervivencia dentro de los pacientes con CR metastático. En nuestra serie, la fórmula leucocitaria parece transmitir que a un mayor número de linfocitos y de polimorfonucleares, tanto el tamaño tumoral como la posibilidad de progresión disminuyen, lo que concuerda con la importancia de la respuesta inmune para "contener" el crecimiento y la progresión del tumor.
La elevación aislada de las fosfatasas alcalinas ofrece resultados controvertidos como valor pronóstico; una vez descartadas las metástasis óseas (hasta un 39\%) mantienen niveles de fosfatasas alcalinas normales ${ }^{3}$ o hepáticas, su elevación se considera un síndrome paraneoplásico que ocurre hasta el $21 \%$ de los casos, pero no tiene relación con el estadio tumoral ni con la posterior evolución tumoral tras tratamiento, aunque su asociación con otros síndromes paraneoplásicos lógicamente marca un peor pronósti$\mathrm{co}^{4}$. En nuestra serie, la elevación de las fosfatasas alcalinas se relacionó con los momentos de progresión de la enfermedad.

Dentro de la analítica estándar, se pueden incluir los reactantes de fase aguda. La PCR es producida por los hepatocitos en presencia de IL-6, estando relacionados los niveles de ambas moléculas en suero ${ }^{10,13}$. En nuestra serie, su elevación coincidió con los momentos de progresión de la enfermedad. En aquellos pacientes con CR metastásico en los que su expresión sérica está elevada, la realización de nefrectomía citorreductora confiere un mejor pronóstico respecto a los pacientes en que ésta no se lleva a cabo por algún motivo. Este mejor pronóstico se refuerza si los niveles de la PCR se normalizan tras la nefrectomía ${ }^{1}$.

El metabolismo férrico ha sido estudiado extensamente en el CR y otros tumores (mama, neuroblastoma y leucemia linfoblástica aguda) en la pasada década. En concreto la ferritina, proteína cuya función es el almacenaje del hierro, puede estar elevada en pacientes con dichos tumores sin un acúmulo paralelo de hierro, debido a que se trate de un reactante de fase aguda, a que esté disminuida su eliminación o bien a que el propio tumor la produzca en exceso. Esta última posibilidad ha sido demostrada por Kirkali et al., tanto en sangre procedente de la vena renal de riñones tumorales como en el propio tumor respecto a parénquima sano ${ }^{6}$. Ello ha dado pie a valorar la expresión de la ferritina como marcador sérico en el CR. Se han publicado diferencias estadísticamente significativas entre los valores de la ferritina en pacientes con CR respecto a un grupo control, su relación con el estadio y el volumen tumoral ${ }^{5}$ y su valor pronóstico en relación a su normalización o no tras la cirugía renal ${ }^{7}$. Entre nuestros pacientes con ferritina inicialmente elevada fue más frecuente la progresión tumoral. 
Distintos marcadores tumorales glicoproteicos o glicolipídicos, derivados de la alteración de la membrana celular debido al proceso oncogénico, han sido descritos en diversos tumores. En un estudio sobre 154 pacientes con $\mathrm{CR}$, estableciendo valores de referencia con voluntarios sanos y limitando el posible artefacto que en los valores de estos marcadores supone la insuficiencia renal, Grankvist et al. observan un valor sérico elevado pre-tratamiento del CA-50, CA-19-9, CA-15-3 y del CA-125, mientras que el antígeno carcinoembrionario (CEA) permanece normal; sin embargo, en el estudio multivariado, tan solo el CA-125, junto con el grado y el estadio tumoral, son relevantes respecto a la supervivencia, proponiendo al CA-125 como marcador pronóstico útil en el $\mathrm{CR}^{9}$. Nuestros resultados coinciden con los de esta serie, mostrando que de todos los marcadores tumorales glicoproteicos, quizá sólo el CA125 ofrezca algún valor pronóstico.

Este mismo grupo estudió previamente la $\beta_{2}$ microglobulina, observando una relación con grado y estadio de sus valores séricos, con valor pronóstico sólo en el estudio univariado y no en el multivariado ${ }^{8}$. En nuestra serie la $\beta_{2}$-microglobulina parece tener una relación estadísticamente significativa con relación a la progresión de la enfermedad. La $\beta 2$-microglobulina es una proteína que actualmente está siendo utilizada como marcador sérico de tumores hematológicos como los linfomas y mielomas; su determinación es rutinaria en cualquier laboratorio y su coste similar al de una determinación de PSA.

El antígeno tisular polipeptídico específico (TPS) sólo se eleva en suero en el 40\% de los pacientes con $\mathrm{CR}$ y no se relaciona claramente con el curso de la enfermedad; sin embargo, sólo los pacientes con enfermedad diseminada que tienen valores normales de este marcador son los que responden a la inmunoterapia, aunque el número de pacientes fue bajo en este estudio ${ }^{4}$. Estos resultados en $\mathrm{CR}$ avanzado se han confirmado en otras series más recientes ${ }^{14}$.

Por último, otro grupo de marcadores séricos extensamente estudiados en el CR son los relacionados con la respuesta inmune. La interleuquina6 (IL-6) es una citoquina multifuncional relacionada en los procesos de respuesta inmune, con la respuesta en fase aguda y la diferenciación de elementos de la serie blanca frente a neoplasias. La IL-6 es la responsable de la mayoría de los síndromes paraneoplásicos que ocurren en el CR. Sus valores séricos en pacientes con CR están elevados frente a la población sana y se relacionan con estadio y grado ${ }^{10}$ y tamaño tumoral ${ }^{13}$ del CR, así como con los reactantes de fase aguda ${ }^{10,13}$; pese a estar relacionados con la supervivencia, su valor pronóstico desaparece frente al del estadio, grado y VSG en el estudio multivariado ${ }^{10}$. Otro grupo de investigadores ha estudiado los valores séricos del receptor soluble de la interleuquina-2, provenientes de los linfocitos activados por la presencia del $\mathrm{CR}$, observando su elevación en pacientes con $\mathrm{CR}$ frente a población normal y mostrando su relación con el estadio, la PCR, la IL-2 y con la supervivencia cáncer-específica ${ }^{11}$, datos que coinciden con los de nuestra serie, donde sus niveles iniciales elevados confieren una mayor posibilidad de progresión y donde se observa su descenso tras el tratamiento de las progresiones. Sin embargo, la fracción sérica del receptor de la IL-6 no es diferente en los pacientes con $\mathrm{CR}^{13}$.

Además de los estudiados en nuestro trabajo, en la literatura se han estudiado otros marcadores séricos. Por ejemplo, existen resultados controvertidos en cuanto al valor pronóstico del TGF- $\beta 1$ en suero ${ }^{15,16}$ y resultados preliminares que ofrecen un valor pronóstico en el estudio multivariado a la proteína ácida sérica inmunosupresiva preoperatoria $^{17}$, aunque estos trabajos deben ser refrendados por series prospectivas por otros autores, ya que, tal y como refleja el excelente trabajo de revisión publicado por Méjean et al. ${ }^{18}$, los factores reconocidos como pronósticos en CR siguen siendo el estadio patológico, el grado, el estado del paciente y el tipo histológico, y los numerosos estudios publicados al respecto, estudiando marcadores séricos, moleculares y en referencia al tratamiento no han podido reconfigurar la clasificación de factores pronósticos en CR, propuesta por el Colegio Americano de Anatomopatólogos en $1995^{19}$.

En otra línea de investigación se ha publicado recientemente un interesante estudio en orina de un panel de genes que normalmente sufren metilaciones en el $\mathrm{CR}$, realizado mediante una PCR que detecta de forma muy sensible la metilación de un solo alelo entre 1.000 no metilados. Esta técnica, que ha ofrecido interesantes resultados en otros fluidos y con otros tumores sólidos (ej. mama/lavado ductal), ha demostrado en orina la metilación de alguno de los 5 genes estudiados (100\% cobertura) con una sensibilidad del 88\% y una especificidad del 100\%, no detectándose en 
voluntarios sanos ni enfermos urológicos no tumorales. Si bien no como seguimiento del paciente operado de un $\mathrm{CR}$, esta técnica, con las posibles mejoras que la selección de otros genes implicados en cada tipo de tumor renal puedan aportar, puede ser que sea un arma diagnóstica importante en el futuro en los pacientes en riesgo de padecer un $\mathrm{CR}$ o bien ayude en la decisión terapéutica ante una masa renal no filiada ${ }^{20}$.

\section{CONCLUSIONES}

Dentro del gran número de marcadores propuestos en la literatura, ninguno ha sido científicamente validado como marcador sérico útil desde el punto de vista pronóstico y de seguimiento en el CR. En nuestro Centro seguiremos determinando el hemograma, el recuento diferencial leucocitario y la bioquímica estándar, la PCR, la ferritina, la $\beta-2$ microglobulina, y el RIL-2 tanto en el momento del diagnóstico, en caso de progresión y cada 6 meses si no se objetiva progresión exclusivamente para su ulterior desarrollo y análisis como marcadores útiles en el seguimiento del CR, desestimando la determinación del resto de marcadores testados.

\section{REFERENCIAS}

1. FUJIKAWA K, MATSUI $\mathrm{Y}$, OKA $\mathrm{H}$, FUKUZAWA $\mathrm{S}$, TAKEUCHI H.: Serum C-reactive protein level and the impact of cytoreductive surgery in patients with metastatic renal cell carcinoma. J Urol 1999; 162: 1934-1937.

2. SEAMAN E, GOLUBOFF ET, ROSS S, SAWCZUK IS.: Association of radionuclide bone scan and serum alkaline phosphatase in patients with metastatic renal cell carcinoma. Urology 1996; 48: 692-695.

3. CHUANG Y, LIN AT, CHEN K, CHANG Y, CHEN M, CHANG LS.: Paraneoplastic elevation of serum alkaline phosphatase in renal cell carcinoma. Incidence and implication on prognosis. J Urol 1997; 158: 1684-1687.

4. HOBARTH K, HALLAS A, KRANER G, AULIZKY W, GOMAHR A, STEINER G, MARBERGER M.: Tissue polypeptide-specific antigen in renal cell carcinoma. Eur Urol 1996; 30: 89-95.

5. PARTIN AW, CRILEY SR, STEINER MS, SSIEH K, SIMONS JW, LUMADUE J, CARTER HB, MARSHALL FF.: Serum ferritin as a clinical marker for renal cell carcinoma: influence of tumour volume. Urology 1995; 45: $211-217$.

6. KIRKALI Z, ESEN AA, KIRKALI G, GÜNER G.: Ferritin: a tumour marker expressed by renal cell carcinoma. Eur Urol 1995; 28: 131-134.

7. ÖZEN H, UYGUR C, SAHIN A, TEKGÜL S, ERGEN A, REMZI D.: Clinical significance of serum ferritin in patients with renal cell carcinoma. Urology 1995; 46: 494-498.

8. RASMUNSON T, GRANKVIST K, LJUNGBERG B.: Serum $\beta 2$-microglobulina and prognosis of patients with renal cell carcinoma. Acta Oncológica 1996; 35: 479-482.
9. GRANKVIST K, LJUNGBERG B, RASMUSON T.: Evaluation of five glycoprotein tumour markers for the prognosis of renal cell carcinoma. Int J Cancer 1997; 74: 233-236.

10. LJUNBERG B, GRANKVIST K, RASMUSON T.: Serum interleukin- 6 in relation to acute phase reactants and survival in patients with renal cell carcinoma. Eur $J$ Cancer 1997; 33: 1794-1798.

11. MATSUMOTO T, FURUKAWA A, SUMIYOSHI Y, AKIYAMA K, KANAYAMA H, KAGAWA S.: Serum levels of soluble interleukin-2 receptor in renal cell carcinoma. Urology 1998; 51: 145-149.

12. CITTERIO G, BERTUZZI A, TRESOLDI M, GALLI L, DI LUCCA G, SCAGLIETTI U, RUGARLI C.: Prognostic factors for survival in metastatic renal cell carcinoma: retrospective analysis from 109 consecutive patients. Eur Urol 1997; 31: 286-291.

13. COSTES V, LIAUTARD J, PICOT MC, ROBERT M, LEQUEUX N, BROCHIER J, BALDET P, ROSSI JF. Expression of the interleukin 6 receptor in primary renal cell carcinoma. J Clin Pathol 1997; 50: 835-840.

14. CHANG CH, WU HC, YEN RF, KAO A, LIN CC, LEE CC.: Tissue polypeptide specific antigen (TPS) as a tumour marker in renal cell carcinoma. Anticancer Res 2002; 22 (5): 2949-2950.

15. WUNDERLICH H, STEINER T, JUNKER U, KNOFEEL B, SCHLINDER A, SCHUBERT J.: Serum transforming growth factor beta-1 in patients with renal carcinoma. J Urol 1997; 157: 1602-1603.

16. HEGELE A, VARGA $Z$, VON KNOBLOCH R, HEIDENREICH A, KROPF HOFMANN R.: TGF- $\beta 1$ in patients with renal cell carcinoma. Urol Res 2002; 30: 126129.

17. MATSUMOTO K, IWAMURA M, MURAMOTO M, SUYAMA K, TABATA K, MINEI S, HIRAI S, BABA S.: Prognostic value of serum immunosuppressive acidic protein in renal cell carcinoma. Nippon Hinyokika Gakkai Zasshi 2002; 93 (4): 548-554.

18. MÉJEAN A, OUDARD S, THIOUNN N.: Prognostic factors of renal cell carcinoma. J Urol 2003; 169: 821827.

19. HENSON DE, FIELDING LP, GRIGNON DJ, PAGE DL, HAMMOND ME, NASH G et al.: College of American Pathologists Conference XXVI on clinical relevance of prognostic markers in solid tumours. Summary. Members of the Cancer Committee. Arch Pathol Lab Med 1995; 119: 1109.

20. BATTAGLI C, UZZO RG, DULAIMI E, IBAÑEZ DE CACERES I, KRASSENSTEIN R, AL-SALEEM T, GREENBERG RE, CAIRNS P.: Promoter hypermethylation of tumour suppressor genes in urine from kidney cancer patients. Cancer Research 2003; 63: 86958699.

Dr. J. Rubio Briones

Servicio de Urología

Instituto Valenciano de Oncología

C/ Profesor Beltrán Báguena, 8

46009 Valencia

(Trabajo recibido el 16 diciembre 2003) 